Revista de Matemática: Teoría y Aplicaciones 1994 1(1) : 31-40

CIMPA - UCR - CCSS ISSN: 1409-2433

\title{
ALGUNAS CONSIDERACIONES SOBRE EL MODELO LINEAL MULTIVARIADO
}

\author{
Jorge Poltronieri Vargas* ${ }^{*}$ Asdrúbal Duarte Esquivel ${ }^{* *}$
}

\begin{abstract}
Resumen
Se trata el modelo lineal general y las pruebas de hipótesis clásicas. En particular se considera el análisis de varianza a dos factores, dándose explicitamente las formas de las matrices asociadas, así como la estadística del cociente de verosimilitud para las hipótesis nulas. También se obtienen en forma explícita las distribuciones de los estimadores, bajo la hipótesis de normalidad.
\end{abstract}

\begin{abstract}
We consider the general linear model with the classical hypothesis. In particular we consider the variance analysis in two factors and we give explicitly the forms of the asocited matrixes as well as the statistics of the likelihood ratio test for the null hypothesis. We obtain in a explicit form the distributions of the estimator under hypothesis of normality.
\end{abstract}

\section{Introducción}

Los resultados teóricos desarrollados en [4] y en [5], se aplican aquí al modelo lineal general multidimensional. Se tratan los resultados clásicos de pruebas de hipótesis, y en particular el análisis de varianza a dos factores.

Consideremos $X_{1}, \ldots, X_{N}$ vectores aleatorios independientes tales que :

$$
X_{\alpha} \sim N\left(\mu_{\alpha}, \Sigma\right) \quad \alpha=1, \ldots, N .
$$

Denotaremos $X=\left(X_{1}, \ldots, X_{N}\right) \sim N(\Gamma, I \otimes \Sigma)$, donde $\Gamma=\left(\mu_{1}, \ldots, \mu_{N}\right)$. Observemos que si $X_{\alpha} \sim N(\mu, \Sigma)$, se tiene que $\Gamma=(\mu, \ldots, \mu)$. Además:

$$
I-\frac{1}{N} J, \text { con } J=\mathbf{1} \mathbf{1}^{\prime}, \quad \mathbf{1}^{\prime}=(1, \ldots, 1),
$$

\footnotetext{
*Escuela de Matemática, Universidad de Costa Rica, 2060 San José, Costa Rica. E-Mail: jpvargas@racsa.co.cr

** Escuela de Matemática, Universidad de Costa Rica, 2060 San José, Costa Rica. E-Mail: aduarte@carari.ucr.ac.cr
} 
es una matriz simétrica idempotente; por lo tanto:

$$
X\left(I-\frac{1}{N} J\right) \sim N\left(0,\left(I-\frac{1}{N} J\right) \otimes \Sigma\right)
$$

pues $\left(I-\frac{1}{N} J\right) \Gamma=0$, y dado que $\operatorname{rang}\left(I-\frac{1}{N} J\right)=N-1$,

$$
X\left(I-\frac{1}{N} J\right) X^{\prime}=\sum_{\alpha=1}^{N}\left(X_{\alpha}-\bar{X}\right)\left(X_{\alpha}-\bar{X}\right)^{\prime} \sim W(\Sigma, N-1),
$$

donde $W(\Sigma, N-1)$ designa la distribusión Wishart de matriz de varianzas $\Sigma$ y $N-1$ grados de libertad.

Por otro lado, si $X_{\alpha} \sim N\left(\Lambda m_{\alpha}, \Sigma\right), \quad \alpha=1, \ldots, N$, son independientes, con $m_{\alpha}$ un vector $r$-dimensional y $H=M M^{\prime}=\sum_{\alpha=1}^{N} m_{\alpha} m_{\alpha}^{\prime}$ no singular, se tiene que $I-M^{\prime} H^{-1} M$ es idempotente. Se sabe que :

$$
\begin{gathered}
X \sim N(\Lambda M, I \otimes \Sigma), \\
X\left(I-M^{\prime} H^{-1} M\right) \sim N\left(0,\left(I-M^{\prime} H^{-1} M\right) \otimes \Sigma\right),
\end{gathered}
$$

puesto que $\Lambda M\left(I-M^{\prime} H^{-1} M\right)=0$ y la forma cuadrática:

$$
X\left(I-M^{\prime} H^{-1} M\right) X^{\prime} \sim W(\Sigma, N-r),
$$

donde $r=$ rang $H$.

\section{El modelo lineal}

Consideremos $X_{1}, \ldots, X_{N}$ variables aleatorias independientes, tales que:

$$
X_{\alpha} \sim N\left(\beta Z_{\alpha}, \Sigma\right), \quad \alpha=1, \ldots, N,
$$

con $Z_{\alpha}$ vector $q$-dimensional, $\beta$ matriz $p \times q$ y $\Sigma$ matriz de covarianza.

Las matrices $\beta$ y $\Sigma$ son desconocidas. Así el modelo se escribe:

$$
X \sim N(\beta Z, I \otimes \Sigma)
$$

donde $Z=\left(Z_{1}, \ldots, Z_{N}\right)$. Los estimadores de máxima verosimilitud son:

$$
\begin{gathered}
\hat{\beta}=X Z^{\prime} A^{-1} \\
N \hat{\Sigma}=\sum_{\alpha=1}^{N}\left(x_{\alpha}-\hat{\beta} Z_{\alpha}\right)\left(x_{\alpha}-\hat{\beta} Z_{\alpha}\right)^{\prime}=\sum_{\alpha=1}^{N} X_{\alpha} X_{\alpha}^{\prime}-\hat{\beta} A \hat{\beta}^{\prime},
\end{gathered}
$$

donde $A=Z Z^{\prime}$, i.e. $\hat{\beta} \sim N\left(\beta, A^{-1} \otimes \Sigma\right), \quad N \hat{\Sigma} \sim W(\Sigma, N-q)$.

Denotaremos un estimador con subíndice $\Omega$ cuando se trate del estimador de máxima verosimilitud de la muestra, y con subíndice $\omega$ cuando se trate del estimador de máxima verosimilitud bajo la hipótesis $H_{0}$. 
Consideremos $Q=X Z^{\prime} A^{-1} Z \sim N\left(\beta Z, Z^{\prime} A^{-1} Z \otimes \Sigma\right)$, pues $Z^{\prime} A^{-1} Z$ es idempotente. Suponemos que $\beta=\left(\beta_{1}, \beta_{2}\right)$ tales que $\beta_{1}$ tiene $q_{1}$ columnas y $\beta_{2}$ tiene $q_{2}$ columnas. Si deseamos considerar la hipótesis $H_{0}: \beta_{1}=\beta_{1}^{*}$, donde $\beta_{1}^{*}$ es una matriz dada, se tiene que:

$$
\hat{\beta}_{1 \Omega} \sim N\left(\beta_{1}, A_{11 \cdot 2} \otimes \Sigma\right),
$$

con

$$
A=\left[\begin{array}{ll}
A_{11} & A_{12} \\
A_{21} & A_{22}
\end{array}\right], A_{11 \cdot 2}=A_{11}-A_{12} A_{22}^{-1} A_{21} .
$$

Así: $\hat{\beta}_{\Omega} \sim N\left(\beta, A^{-1} \otimes \Sigma\right), \quad\left(\hat{\beta}_{1 \Omega}-\beta_{1}^{*}\right) A_{11 \cdot 2}\left(\hat{\beta}_{1 \Omega}-\beta_{1}^{*}\right) \sim W\left(\Sigma, q_{1}\right), \operatorname{con} q_{1}=\operatorname{rang}\left(A_{11 \cdot 2}\right)$.

Sea $Y=X-\beta_{1}^{*} Z_{1} \sim N\left(\beta_{2} Z_{2}, I \otimes \Sigma\right)$, entonces:

$$
\hat{\beta}_{2 \omega}=Y\left(Z_{2}^{\prime} A_{22}^{-1}\right)=\left(X Z_{2}^{\prime}-\beta_{1}^{*} A_{12}\right) A_{22}^{-1},
$$

donde:

$$
Z=\left[\begin{array}{l}
Z_{1} \\
Z_{2}
\end{array}\right], A_{11}=Z_{1} Z_{1}^{\prime}, A_{22}=Z_{2} Z_{2}^{\prime}, A_{12}=Z_{1} Z_{2}^{\prime}
$$

Bajo la hipótesis $H_{0}$ se tiene:

$$
\begin{gathered}
\hat{\beta}_{2 \omega} \sim N\left(\beta_{2}, A_{22}^{-1} \otimes \Sigma\right), \\
N \hat{\Sigma}_{\omega}=\sum_{\alpha=1}^{N} Y_{\alpha} Y_{\alpha}^{\prime}-\hat{\beta}_{2 \omega} A_{22} \hat{\beta}_{2 \omega}^{\prime}=Y\left(I-Z_{2} A_{22}^{-1} Z_{2}^{\prime}\right) Y^{\prime} \sim W\left(\Sigma, N-q_{2}\right),
\end{gathered}
$$

pues $I-Z_{2} A_{22}^{-1} Z_{2}^{\prime}$ es idempotente de rango $N-q_{2}\left(q=q_{1}+q_{2}\right)$.

Para probar la hipótesis $H_{0}: \beta_{1}=\beta_{1}^{*}$ se utiliza la estadística:

$$
U=\frac{\left|N \hat{\Sigma}_{\Omega}\right|}{\left|N \hat{\Sigma}_{\omega}\right|}
$$

y se compara con $U_{p, q_{1}, N-q}(\alpha)$, donde $\alpha$ es el nivel. La cantidad $\left|N \hat{\Sigma}_{\Omega}\right|$ denota el determinante de $N \hat{\Sigma}_{\Omega}$.

\section{Prueba de igualdad de medias}

Consideremos $Y_{\alpha}^{(i)} \sim N\left(\mu^{(i)}, \Sigma\right) \quad \alpha=1, \ldots, N_{i}, \quad i=1, \ldots, q$, observaciones de $q$ poblaciones de igual matriz de covarianza. Sea $H_{0}$ la hipótesis de igualdad de medias para las $q$ poblaciones, i.e. $H_{0}: \mu^{(1)}=\cdots=\mu^{(q)}$. Se define $X_{N_{1}+\cdots+N_{i-1}+k}=Y_{k}^{(i+1)}$ y tenemos:

$$
\begin{gathered}
\Gamma=\left(\mu^{(1)}, \ldots, \mu^{(1)}, \ldots, \mu^{(q)}, \ldots, \mu^{(q)}\right), \\
X=\left(Y_{1}^{(1)}, \ldots, Y_{N_{1}}^{(1)}, \ldots, Y_{1}^{(q)}, \ldots, Y_{N_{q}}^{(q)}\right),
\end{gathered}
$$

por lo que:

$$
X \sim N(\Gamma, I \otimes \Sigma), \quad N=\sum_{i=1}^{q} N_{i} .
$$


Se utiliza el modelo lineal, introduciendo las variables $Z_{\alpha}, \alpha=1, \ldots, N$, de la forma:

$$
Z=\left(Z_{1}, \ldots, Z_{N}\right)=\left[\begin{array}{ccccccc}
1 & \ldots & 1 & \ldots & 0 & \ldots & 0 \\
0 & \ldots & 0 & \ldots & 0 & \ldots & 0 \\
\vdots & \ddots & \vdots & \ldots & \vdots & \ddots & \vdots \\
0 & \ldots & 0 & \ldots & 1 & \ldots & 1 \\
1 & \ldots & 1 & \ldots & 1 & \ldots & 1
\end{array}\right]
$$

y se define $\beta=\left(\beta_{1}, \beta_{2}\right)$ por:

$$
\begin{gathered}
\beta_{1}=\left(\mu^{(1)}-\mu^{(q)}, \ldots, \mu^{(q-1)}-\mu^{(q)}\right), \\
\beta_{2}=\mu^{(q)} .
\end{gathered}
$$

Así se obtiene que: $X_{\alpha} \sim N\left(\beta Z_{\alpha}, \Sigma\right) \quad \alpha=1, \ldots, N$. La hipótesis $H_{0}$ es: $\beta_{1}=0$. La matriz $A=Z Z^{\prime}$ es:

y

$$
\left[\begin{array}{cccc}
N_{1} & \cdots & 0 & N_{1} \\
\vdots & \ddots & \vdots & \vdots \\
0 & \cdots & N_{q-1} & N_{q-1} \\
N_{1} & \cdots & N_{q-1} & N
\end{array}\right]
$$

$$
\begin{gathered}
C=X Z^{\prime}=\left(C_{1}, C_{2}\right)=\left(\sum_{\alpha} Y_{\alpha}^{(1)}, \ldots, \sum_{\alpha} Y_{\alpha}^{(q-1)}, \sum_{\alpha i} Y_{\alpha}^{(i)}\right), \\
A_{22}=N, \quad C_{2}=\sum_{\alpha i} Y_{\alpha}^{(i)}, \\
\hat{\beta}_{2 \omega}=C_{2} A_{22}^{-1}=\frac{1}{N} \sum_{\alpha i} Y_{\alpha}^{(i)}=\bar{Y} \sim N\left(\beta_{2}, \frac{1}{N} \Sigma\right), \\
N \hat{\Sigma}_{\omega}=\sum_{\alpha i} Y_{\alpha}^{(i)} Y_{\alpha}^{(i) \prime}-\beta_{2 \omega} A_{22} \beta_{2 \omega}^{\prime}=\sum_{\alpha i}\left(Y_{\alpha}^{(i)}-\bar{Y}\right)\left(Y_{\alpha}^{(i)}-\bar{Y}\right)^{\prime} \\
=Y\left(I-\frac{1}{N} J\right) Y^{\prime} \sim W(\Sigma, N-1),
\end{gathered}
$$

pues bajo la hipótesis $H_{0}$ se tiene $\Gamma\left(I-\frac{1}{N} J\right)=0$.

El estimador $\hat{\beta}_{\Omega}=C A^{-1}=\left(\bar{Y}^{(1)}-\bar{Y}^{(q)}, \ldots, \bar{Y}^{(q-1)}-\bar{Y}^{(q)}, \bar{Y}^{(q)}\right)$, donde $\bar{Y}^{(i)}=\frac{1}{N_{i}} \sum_{\alpha=1}^{N_{i}} Y_{\alpha}^{(i)}$. Además:

$$
\begin{gathered}
C A^{-1} C^{\prime}=\sum_{i=1}^{q} N_{i} \bar{Y}^{(i)} \bar{Y}^{(i) \prime}, \\
N \hat{\Sigma}_{\Omega}=\sum_{\alpha i}\left(Y_{\alpha}^{(i)}-\bar{Y}^{(i)}\right)\left(Y_{\alpha}^{(i)}-\bar{Y}^{(i)}\right)^{\prime} .
\end{gathered}
$$

Sea $H$ la matriz definida por:

$$
H=\left[\begin{array}{ccc}
J_{1} & \cdots & 0 \\
\vdots & \ddots & \vdots \\
0 & \cdots & J_{q}
\end{array}\right]
$$


con $J_{i}=\frac{1}{N_{i}} \mathbf{1}_{N_{i}} \mathbf{1}_{N_{i}}^{\prime}$. Así definida $H$ es idempotente, de rango $q$ y además :

$$
Y H=\left(\bar{Y}^{(1)}, \ldots, \bar{Y}^{(1)}, \ldots, \bar{Y}^{(q)}, \ldots, \bar{Y}^{(q)}\right) \sim N(\Gamma, H \otimes \Sigma),
$$

pues $\Gamma H=\Gamma$. Por otro lado:

$$
N \hat{\Sigma}_{\Omega}=\sum_{\alpha i}\left(Y_{\alpha}^{(i)}-\bar{Y}^{(i)}\right)\left(Y_{\alpha}^{(i)}-\bar{Y}^{(i)}\right)^{\prime} \sim W(\Sigma, N-q) .
$$

Si $H_{0}$ es la verdadera hipótesis, la estadística: $U=\left|N \hat{\Sigma}_{\Omega}\right| /\left|N \hat{\Sigma}_{\omega}\right|$ se distribuye como una $U_{p, q-1, N-q}$.

Observemos que :

- $N \hat{\Sigma}_{\omega}-N \hat{\Sigma}_{\Omega}=Y\left(H-\frac{1}{N} J\right) Y^{\prime} \sim W(\Sigma, q-1)$.

- $N \hat{\Sigma}_{\omega} /(N-q)$ es un estimador sin sesgo de $\Sigma$, si $H_{0}$ es verdadera.

\section{Análisis de varianza}

El caso analizado anteriormente puede ser considerado como un análisis de varianza a un factor. Vamos a desarrollar aquí el caso de dos factores con una observación por celda.

Sea $Y_{i j} i=1, \ldots, r ; j=1, \ldots, c$, una variable aleatoria $p$-dimensional tal que:

$$
Y_{i j}=\mu+\lambda_{i}+\nu_{j}+E_{i j},
$$

donde $\operatorname{los} E_{i j} \sim N(0, \Sigma)$ son independientes; $\mu, \lambda_{i}, \nu_{j}$ son vectores tales que:

$$
\sum_{i=1}^{r} \lambda_{i}=0, \sum_{j=1}^{c} \nu_{j}=0 .
$$

El modelo se escribe :

$$
Y=\beta Z+E,
$$

donde:

$$
\begin{gathered}
Y=\left(Y_{11}, \ldots, Y_{1 c}, \ldots, Y_{r 1}, \ldots, Y_{r c}\right), \\
\beta=\left(\mu, \lambda_{1}, \ldots, \lambda_{r}, \nu_{1}, \ldots, \nu_{c}\right), \\
E=\left(E_{11}, \ldots, E_{1 c}, \ldots, E_{r 1}, \ldots, E_{r c}\right),
\end{gathered}
$$

y

$$
Z=\left[\begin{array}{ccccccc}
1 & \ldots & 1 & \ldots & 1 & \ldots & 1 \\
1 & \ldots & 1 & \ldots & 0 & \ldots & 1 \\
\vdots & \ddots & \vdots & \ddots & \vdots & \ddots & \vdots \\
0 & \ldots & 0 & \ldots & 1 & \ldots & 1 \\
1 & \ldots & 0 & \ldots & 1 & \ldots & 0 \\
\vdots & \ddots & \vdots & \ddots & \vdots & \ddots & \vdots \\
0 & \ldots & 1 & \ldots & 0 & \ldots & 1
\end{array}\right]
$$


Sea $\bar{Y}_{. .}=\frac{1}{r c} \sum_{i j} Y_{i j}, \quad \bar{Y}_{i .}=\frac{1}{c} \sum_{j} Y_{i j}, \quad \bar{Y}_{\cdot j}=\frac{1}{r} \sum_{i} Y_{i j}$, entonces:

$$
\begin{gathered}
\sum_{i j}\left(Y_{i j}-\bar{Y}_{. .}\right)\left(Y_{i j}-\bar{Y}_{. .}\right)^{\prime}=\sum_{i j}\left(Y_{i j}-\bar{Y}_{i \cdot}-\bar{Y}_{. j}+\bar{Y}_{. .}\right)\left(Y_{i j}-\bar{Y}_{i \cdot-}-\bar{Y}_{. j}+\bar{Y}_{. .}\right)^{\prime} \\
+c \sum_{i}\left(\bar{Y}_{i .}-\bar{Y}_{. .}\right)\left(\bar{Y}_{i .}-\bar{Y}_{. .}\right)^{\prime}+r \sum_{j}\left(\bar{Y}_{. j}-\bar{Y}_{. .}\right)\left(\bar{Y}_{. j}-\bar{Y}_{. .}\right)^{\prime} .
\end{gathered}
$$

Vamos a determinar las matrices asociadas a cada una de las formas cuadráticas.

Sean las matrices:

$$
H_{1}=\frac{1}{c}\left[\begin{array}{ccc}
J & \cdots & 0 \\
\vdots & \ddots & \vdots \\
0 & \cdots & J
\end{array}\right]
$$

con $J=\mathbf{1}_{c} \mathbf{1}_{c}^{\prime}$, i.e. $H_{1}$ tiene $r$ matrices $J$ en la diagonal;

$$
\begin{gathered}
L=\frac{1}{r c} \mathbf{1}_{r c} \mathbf{1}_{r c}^{\prime}, \\
H_{2}=\frac{1}{r}\left[\begin{array}{ccc}
P_{1} & \ldots & P_{c} \\
\vdots & \ddots & \vdots \\
P_{1} & \ldots & P_{c}
\end{array}\right] \text { con } P_{i}=\left[\begin{array}{ccc}
0 & \ldots & 0 \\
\vdots & \ddots & \vdots \\
1 & \ldots & 1 \\
\vdots & \ddots & \vdots \\
0 & \ldots & 0
\end{array}\right],
\end{gathered}
$$

donde el vector $(1, \ldots, 1)$ está situado en la posición $i$. La matriz $P_{i}$ es $r \times r$ y $P_{i} P_{j}=P_{i}$. Las matrices $H_{1}, L, H_{2}$ son idempotentes de rangos respectivos $r, 1, c$. Así tenemos:

$$
\begin{aligned}
Y L & =\left(\bar{Y}_{. .}, \ldots, \bar{Y}_{. .}, \ldots, \bar{Y}_{. .}, \ldots, \bar{Y}_{. .}\right) \\
Y H_{1} & =\left(\bar{Y}_{1 .}, \ldots, \bar{Y}_{1 .}, \ldots, \bar{Y}_{r .}, \ldots, \bar{Y}_{r .}\right) \\
Y H_{2} & =\left(\bar{Y}_{\cdot 1}, \ldots, \bar{Y}_{. c}, \ldots, \bar{Y}_{\cdot 1}, \ldots, \bar{Y}_{. c}\right) .
\end{aligned}
$$

Sabemos que $H_{1}-L$ es idempotente de rango $r-1, \mathrm{y}$

$$
Y\left(H_{1}-L\right) \sim N\left(\Gamma\left(H_{1}-L\right),\left(H_{1}-L\right) \otimes \Sigma\right),
$$

donde $\Gamma\left(H_{1}-L\right)=\left(\lambda_{1}, \ldots, \lambda_{1}, \ldots, \lambda_{r}, \ldots, \lambda_{r}\right)$.

La forma cuadrática:

$$
B_{1}=Y\left(H_{1}-L\right) Y^{\prime}=c \sum_{i=1}^{r}\left(\bar{Y}_{i .}-\bar{Y}_{. .}\right)\left(\bar{Y}_{i .}-\bar{Y}_{. .}\right)^{\prime} \sim W(\Sigma, r-1, \tau),
$$

donde el parámetro de decentraje $\tau=\Gamma\left(H_{1}-L\right) \Gamma^{\prime}=c \sum_{i=1}^{r} \lambda_{i} \lambda_{i}^{\prime}$.

La matriz $\mathrm{H}_{2}-L$ es idempotente de rango $c-1$, y

$$
B_{2}=Y\left(H_{2}-L\right) Y^{\prime}=r \sum_{j=1}^{c}\left(\bar{Y}_{. j}-\bar{Y}_{. .}\right)\left(\bar{Y}_{. j}-\bar{Y}_{. .}\right)^{\prime} \sim W(\Sigma, c-1, \tau),
$$


donde el parámetro de decentraje $\tau=\Gamma\left(H_{2}-L\right) \Gamma^{\prime}=r \sum_{j=1}^{c} \nu_{j} \nu_{j}^{\prime}$.

La matriz $I-H_{1}-H_{2}+L$ es idempotente de rango $(r-1)(c-1)$ :

$$
Y\left(I-H_{1}-H_{2}+L\right) \sim N\left(0,\left(I-H_{1}-H_{2}+L\right) \otimes \Sigma\right),
$$

pues $\Gamma\left(I-H_{1}-H_{2}+L\right)=0$, por lo que:

$$
\begin{gathered}
A=Y\left(I-H_{1}-H_{2}+L\right) Y^{\prime}= \\
\sum_{i j}\left(Y_{i j}-\bar{Y}_{i .}-\bar{Y}_{. j}+\bar{Y}_{. .}\right)\left(Y_{i j}-\bar{Y}_{i .}-\bar{Y}_{. j}+\bar{Y}_{. .}\right)^{\prime} \sim W(\Sigma,(r-1)(c-1)) .
\end{gathered}
$$

Notemos que las formas cuadráticas $A, B_{1}, B_{2}$ son independientes, pues el producto de las matrices asociadas es cero $\left(H_{1} H_{2}=H_{1} L=H_{2} L=L L=L\right)$, y que $B_{1}$ y $B_{2}$ son distribuciones Wishart decentradas.

Si se considera la hipótesis $H_{0}: \lambda_{i}=0, i=1, \ldots, r$, es decir, el efecto del primer factor es nulo, se tiene que $\Gamma\left(H_{1}-L\right)=0$. Bajo la hipótesis $H_{0}$, la estadística:

$$
B_{1}=Y\left(H_{1}-L\right) Y^{\prime} \sim W(\Sigma, r-1) .
$$

De esta manera la estadística:

$$
U=\frac{|A|}{\left|A+B_{1}\right|} \sim U_{p, r-1,(r-1)(c-1)} .
$$

Si se considera la hipótesis $H_{0}: \nu_{j}=0, j=1, \ldots, c$, es decir, el efecto del segundo factor es nulo, se tiene que $\Gamma\left(H_{2}-L\right)=0$, y la estadística:

$$
B_{2}=Y\left(H_{2}-L\right) Y^{\prime} \sim W(\Sigma, c-1) .
$$

Así la estadística:

$$
U=\frac{|A|}{\left|A+B_{2}\right|} \sim U_{p, c-1,(r-1)(c-1)} .
$$

En los dos casos se rechaza la hipótesis $H_{0}$ si :

$$
U \leq U_{p, m,(r-1)(c-1)}(\alpha)
$$

para $m=r-1$, o $m=c-1$.

Este resultado se generaliza fácilmente en el caso de $n$ observaciones por celda, $n>1$.

\section{Aplicaciones}

En está sección vamos a considerar algunos ejemplos., lo cuales nos ayudarán a comprender, la utilidad de la teoría desarrollada en este trabajo.

1. Primeramente consideraremos un estudio realizado por Bernard (1935) (ver [1]), en el cual realiza $4(=p)$ medidas sobre cráneos egipcios, correspondiendo a $4(=p)$ poblaciones: 
Predinástica $(i=1)$, sexta a undésima dinastía $(i=2)$, duodécima y decimotercera dinastía $(i=3)$, y la dinastía Ptoloméica $(i=4)$.

El número de observaciones por poblaciones $N_{1}=91, N_{2}=162, N_{3}=70, N_{4}=75$. La hipótesis $H_{0}$ que consideramos es que las cuatro poblaciones son iguales, es decir, las medias de las poblaciones son idénticas $\mu^{(1)}=\mu^{(2)}=\mu^{(3)}=\mu^{(4)}$.

Los datos sumarizados son:

$$
\begin{gathered}
\left(\bar{Y}^{(1)}, \bar{Y}^{(2)}, \bar{Y}^{(3)}, \bar{Y}^{(4)}\right)=\left(\begin{array}{cccc}
133,582 & 134,265 & 134,371 & 135,306 \\
98,307 & 96,462 & 95,857 & 95,040 \\
50,835 & 51,148 & 50,100 & 52,093 \\
133,000 & 134,882 & 133,642 & 131,466
\end{array}\right) \\
N \Sigma_{\Omega}=\left(\begin{array}{cccc}
9661,997 & 445,573 & 11130,623 & 214,584 \\
445,573 & 9073,115 & 1239,211 & 2255,812 \\
1130,623 & 1239,211 & 3938,320 & 1271,054 \\
2148,584 & 2255,812 & 1271,054 & 8741,508
\end{array}\right) .
\end{gathered}
$$

De los datos tenemos:

$$
N \hat{\Sigma}_{\omega}=\left(\begin{array}{cccc}
9785,178 & 214,197 & 1217,929 & 2019,820 \\
214,197 & 9559,460 & 1131,716 & 2381,126 \\
2117,929 & 1131,716 & 4088,731 & 1133,473 \\
2019,820 & 2381,126 & 1133,473 & 9382,242
\end{array}\right) .
$$

La estadística está dada por:

$$
U=\frac{\left|N \widehat{\Sigma}_{\Omega}\right|}{\left|N \hat{\Sigma}_{\omega}\right|}=0,8214344,
$$

$N=398, n=394, p=4, q=4$. Como $n$ es grande, aproximamos $-m \log U_{4,3,394}$ con $\chi_{12}^{2}$, cuando la hipótesis $H_{0}$ es cierta. Así $-m \log U=77,30$ y como $\chi_{12}^{2}(0,01)=26,2$, se rechaza la hipótesis $H_{0}$, es decir, hay diferencias significativas entre las poblaciones.

Para el análisis de varianza utilizamos un ejemplo discutido por Anderson(1958). En este ejemplo, se considera como primera componente del vector de observaciones el rendimiento de un campo de cebada; la segunda componente son las mismas medidas hechas al año siguiente $(p=2)$. Los datos aparecen en la tabla siguiente. Los índices columna indican las variedades de cebada y las filas indican las localidades.

Se considera el modelo a dos factores, donde el primer factor es la variedad con $r=5$ niveles, y el segundo factor es la localidad con $c=6$ niveles. La hipótesis $H_{0}$ que se considera, es que el efecto debido a la variedad es nulo, es decir, dentro del modelo:

$$
Y_{i j}=\mu+\lambda_{i}+\nu_{j}+E_{i j}
$$

$\operatorname{los} \lambda_{i}=0, \quad i=1, \ldots, r=5$. En otras palabras, las observaciones se explican por medio de un valor general $\mu \mathrm{y}$ el efecto debido a la localidad $\mu_{j}, \quad j=1, \ldots, c=6$.

Así

$$
\sum_{i, j} Y_{i j} Y_{i j}^{\prime}=\left(\begin{array}{ll}
380944 & 315381 \\
315381 & 277625
\end{array}\right),
$$




\begin{tabular}{|c|ccccc|}
\hline & \multicolumn{5}{|c|}{ Variedades } \\
Localidad & $\mathrm{M}$ & $\mathrm{S}$ & $\mathrm{V}$ & $\mathrm{T}$ & $\mathrm{P}$ \\
\hline UF & 81 & 105 & 120 & 110 & 98 \\
& 81 & 82 & 80 & 87 & 84 \\
$\mathrm{~W}$ & 147 & 142 & 151 & 192 & 146 \\
& 100 & 116 & 112 & 148 & 108 \\
& & & & & \\
$\mathrm{M}$ & 82 & 77 & 78 & 131 & 90 \\
& 103 & 105 & 117 & 140 & 130 \\
$\mathrm{C}$ & 120 & 121 & 124 & 141 & 125 \\
& 99 & 62 & 96 & 126 & 76 \\
$\mathrm{GR}$ & 99 & 89 & 69 & 89 & 104 \\
& 66 & 50 & 97 & 62 & 80 \\
& & & & & \\
$\mathrm{D}$ & 87 & 77 & 79 & 102 & 96 \\
& 68 & 67 & 67 & 92 & 94 \\
\hline
\end{tabular}

Cuadro 1: Variedades de cebada por cada localidad

$$
\begin{aligned}
& \sum_{j}\left(6 \bar{Y}_{i \cdot}\right)\left(6 \bar{Y}_{\cdot j}\right)^{\prime}=\left(\begin{array}{ll}
2157924 & 1844346 \\
1844346 & 1579583
\end{array}\right), \\
& \sum_{i}\left(5 \bar{Y}_{i \cdot}\right)\left(5 \bar{Y}_{i .}\right)^{\prime}=\left(\begin{array}{ll}
1874386 & 1560145 \\
1560145 & 1353727
\end{array}\right), \\
& \left(30 \bar{Y}_{. .}\right)\left(30 \bar{Y}_{. .}\right)=\left(\begin{array}{cc}
10705984 & 9145240 \\
9145240 & 7812025
\end{array}\right) .
\end{aligned}
$$

La suma de los cuadrados de los errores:

$$
\begin{gathered}
A=\left(\begin{array}{cc}
3279 & 802 \\
802 & 4017
\end{array}\right), \\
5 \sum_{i}\left(\bar{Y}_{i .}-\bar{Y}_{. .}\right)\left(\bar{Y}_{i}-\bar{Y}_{. .}\right)^{\prime}=\left(\begin{array}{cc}
18011 & 7188 \\
7188 & 10345
\end{array}\right), \\
B=\left(\begin{array}{cc}
2788 & 2550 \\
2550 & 2863
\end{array}\right) .
\end{gathered}
$$

La estadística para la hipótesis $H_{0}$ es:

$$
U=\frac{|A|}{|A+B|}=0,4107 .
$$


Este resultado se compara con $U_{2,4,20}(0,05)$, o bien se compara con el valor:

$$
\frac{1-\sqrt{0,4017}}{\sqrt{0,4017}} \cdot \frac{19}{4}=2,66
$$

con un $F_{8,38}(0,05)=2,18$ lo que indica que hay diferencias entre variedades, con un error del $5 \%$.

\section{Referencias}

[1] T.W. Anderson (1958) An introduction to multivariate statistical analysis. J. Wiley, N.Y.

[2] J.R. Barra (1971) Notions fondamentales de statistique mathématique. Dunod, Paris.

[3] H. Muirhead (1982) Aspects of multivariate statistical theory. J. Wiley, N.Y.

[4] J. Poltronieri (1988) Estudio de formas cuadráticas en el caso multivariado. In: IV Simposio de Métodos Matemáticos Aplicados a las Ciencias Ed. U.C.R.

[5] J. Poltronieri (1988) Formas cuadráticas y formas lineales en estadística multivariada. In: IV Simposio de Métodos Matemáticos Aplicados a las Ciencias Ed. U.C.R.

[6] K. Takeuchi, H. Yanai, B.N. Mukherjee (1984) The foundations of multivariate analysis. Wiley Eastern Limited.

[7] M. Tenenhaus, F. Young (1987) An analysis and synthesis of multiple correspondance analysis, optimal scaling, dual scaling, homogenity analysis and other methods for quantifying categorical multivariate data, Psychometrica 50(1), pp. 91-119. 